Technical report

\title{
Energy absorption and exposure build-up factors in hydroxyapatite
}

\author{
H.C. Manjunatha ${ }^{\mathrm{a}, *}$, B. Rudraswamy ${ }^{\mathrm{b}}$ \\ a Department of Physics, Government College for Women, Kolar 563101, Karnataka, India \\ ${ }^{\mathrm{b}}$ Department of Physics, Bangalore University, Bangalore 560056, Karnataka, India
}

\section{A R T I C L E I N F O}

\section{Article history:}

Received 26 November 2011

Received in revised form

1 February 2012

Accepted 2 February 2012

\section{Keywords:}

Effective atomic number

Electron density

Hydroxyapatite

\begin{abstract}
A B S T R A C T
The effective atomic number $\left(Z_{\text {eff }}\right)$ and electron density $\left(N_{e l}\right)$ of hydroxyapatite $(\mathrm{HA})$ and cortical bone have been computed for total photon interaction in the wide energy range of $1 \mathrm{keV}-100 \mathrm{GeV}$ using WinXCom. The variations of effective atomic number and electron density with energy of HA are compared with that of cortical bone. GP. fitting method has been used to compute energy absorption and exposure build-up factor of HA for wide energy range $(0.015 \mathrm{MeV}-15 \mathrm{MeV})$ up to the penetration depth of 40mean free path. The computed absorption build-up factor is used to estimate specific absorbed fraction of energy $(\Phi)$ and relative dose of photon in HA. Build-up factor increases with increase of penetration depth. The results of the present paper will also help in estimating safe dose levels for radiotherapy patients and also will be useful in dosimetry and diagnostics.
\end{abstract}

(c) 2012 Elsevier Ltd. All rights reserved.

\section{Introduction}

Gamma and X-radiation are widely used in medical imaging and radiation therapy. The user of radioisotopes must have knowledge about how radiation interacts with matter, especially with the human body, because when photons enter the medium/body, they degrade their energy and build-up in the medium, giving rise to secondary radiation which can be estimated by a factor which is called the 'build-up factor'. Calculations of the energy absorbed in a medium include not only the contribution of the uncollided photons from the source, but must also include the contributions from collided and secondary photons. In practice, this is done by multiplying the contribution of the uncollided photons by the energy absorption build-up factor. The energy absorption build-up factor is also defined as the build-up factor in which the quantity of interest is the absorbed or deposited energy in the interacting material and the detector response function is that of absorption in the interacting medium. Whereas the exposure build-up factor is defined as that build-up factor in which the quantity of interest is the exposure and the detector response function is that of absorption in air.

Singh et al. (2008) studied the variation of energy absorption build-up factors with incident photon energy and penetration depth for some solvents. In this study, the exposure build-up factors are computed on the basis of $Z_{e q}$ which is calculated using the following interpolation formula:

\footnotetext{
* Corresponding author.

E-mail address: manjunathhc@rediffmail.com (H.C. Manjunatha).
}

$Z_{\text {eq }}=\frac{Z_{1}\left(\log R_{2}-\log R\right)+Z_{2}\left(\log R-\log R_{1}\right)}{\left(\log R_{2}-\log R_{1}\right)}$

where $Z_{1}$ and $Z_{2}$ are the elemental atomic numbers corresponding to the ratios $\left(\mu_{\text {comp }} / \mu_{\text {total }}\right) R_{1}$ and $R_{2}$ respectively. $R$ is the ratio for the chosen biological sample at particular energy. Then the GP fitting parameters were computed by interpolation method. The generated energy absorption build-up factor data was studied as a function of penetration depth and incident photon energy. Previous workers [Singh et al. (2008)] used the equivalent atomic numbers, $Z_{e q}$ (which is obtained from interpolation method) to compute the build-up factors. The variation of $Z_{e q}$ with photon energy is not similar to the variation of effective atomic number $\left(Z_{e f f}\right)$. Hence this computed build-up factors may not be accurate. Manjunatha and Rudraswamy (2011a) computed the exposure build-up factors in the different regions of teeth enamel and dentin based on the accurate values of effective atomic number $\left(Z_{e f f}\right)$. Manjunatha and Rudraswamy (2011b) studied the specific fraction of absorbed energies in the energy range $0.015-15 \mathrm{MeV}$. The build-up factor data were computed by different codes such as PALLAS-PL [Takeuchi (1973)], RADHEAT-V4 [Yamano et al. (1989)], ADJMOM1 [Simmons (1973)] and ASFIT [Gopinath and Samthanam, 1971]. American National Standards ANSI/ANS 6.4.3 (1991) used GP fitting method and provided build-up factor data for 23 elements, water, air and concrete at 25 standard energies in the energy range 0.015-15 MeV with suitable interval up to the penetration depth of 40mean free paths. The ANSI/ANS 6.4 .3 (1991) standard for buildup factors has been administratively withdrawn, but work is in progress for updating this standard which is much used. For the time being there are no new reference data for build-up factors. 
Meanwhile, it should be all right to use the ANSI/ANS 6.4 .3 (1991) standard, since the possible discrepancies are expected to be small for the low- $Z$ materials [(Ryman et al. (2008) and Ruggieri and Sanders (2008)]. The geometric progression (GP) fitting seems to reproduce the build-up factors with better accuracy when compared with other available approximations such as Berger, Taylor and three exponential [Harima (1993) and Harima et al. (1986)]. The absolute value of the maximum deviation of buildup factors in the GP fitting is within $0.5-3 \%$, in the threeexponential approach it is within 0.4-9.3\%, in the Berger approach it is within $0.9-42.7 \%$, and in the Taylor approximation it is within $0.4-53.2 \%$ (Harima, 1993). Recently, Asano and Sakamoto (2007) have compared their build-up factors of two typical heavy concretes evaluated by using Monte Carlo simulations code (EGS4) with ANSI/ANS-6.4.3 standard reference database, and concluded that there is good agreement between both, except occasional slight differences. These differences may be due to the fact that ANSI/ANS data is based on the calculations using the moment method [Eisenhauer and Simmons (1975)] with parallel beam source and the Monte Carlo code EGS4 with isotropic emission source. Shimizu et al. (2004) have reported that when using the invariant embedding, GP fitting and Monte Carlo methods agree well for 18 low- $Z$ materials with small discrepancies. All the materials used in the present study consist of low- $Z$ materials.

Hydroxyapatite (HA) is the main mineral constituent of human bone, and also used clinically for many years. It has good biocompatibility in bone contact as its chemical composition is similar to that of bone material. The primary purpose of tissue engineering is repair, regeneration, and reconstruction of lost, damaged or degenerative tissues. Bone tissue itself shows an excellent ability of regeneration, but for big bony defect, healing process is difficult, so that bone grafts are required. At this point, it is very crucial to match the osteoconductive properties of porous ceramic scaffold in one side with the osteoinductive or osteogenic properties of living bone cells in the other side. Theoretically, a degradation rate of the implant similar to the rate of tissue formation is expected. In bone tissue engineering, Hydroxyapatite (HA) has been applied as filling material for defects and augmentation, artificial bone graft material, and prosthesis revision surgery. Its high surface area leads to excellent osteoconductivity and resorbability providing fast bone in growth (Frieb and Warner, 2002; Agarwala and Bhagawat, 2005; Sopyan et al., 2007). Their chemical composition is close to the mineral phase of bone and it is an origin of their excellent biocompatibility to bone tissue. This meets the requirement of any materials designed for bone repair and augmentation (Hench, 1998). The benefits of HA as bone graft are predominantly its safety, biocompatibility and osteoconductivity so that it can be used as a substitution biomaterial for bone in many indications clinically (Damien and Revell, 2004).

Porous HA has been applied for cell loading (Ohgushi and Caplan, 1999; Banfi et al., 2000; Aoki et al., 2004), drug releasing agents (Krajewski et al., 2000; Byrne and Deasy, 2002), chromatography analysis (United States Patent 6210715, 2001), and the most extensively for hard tissue scaffolds (Moore et al., 2001; Muraglia et al., 2000). In drug delivery systems, it has been recognized that, a system for the slow, local and continuous release of drugs would be a decided advantage for the treatment of many ailments. One of potential candidates for such controlled drug delivery systems is porous ceramics; much attention has been paid to porous HA. Owing to their physicochemical and biological properties, porous HAs have been proven as a potential candidate for bone drug delivery system (Komlev et al., 2003). In the present work an attempt has been made to compute energy absorption and exposure build-up factors of $\mathrm{HA}$ for wide energy range $(0.015 \mathrm{MeV}-15 \mathrm{MeV})$ up to the penetration depth of 40mean free path using GP fitting method. Such data will be prime importance in accurate dose calculations which is useful in medical dosimetry.

\section{Present work}

The computations of build-up factor have been divided into three parts, which are as follows.

\subsection{Computation of effective atomic number $\left(Z_{\text {eff }}\right)$ and effective electron density $\left(N_{e l}\right)$}

For computation of $Z_{e f f}$, firstly the values of mass attenuation coefficients were computed from WinXCom computer program (Gerward et al., 2004). $Z_{e f}$ and $N_{e l}$ can be computed from the following equations:

$Z_{\text {eff }}=\frac{\left(\frac{\left(\frac{\mu}{\rho}\right)_{\text {bio }} \sum_{i} n_{i} A_{i}}{\sum_{i} n_{i}}\right)}{\left(\sum_{i}\left(\frac{f_{i} A_{i}}{Z_{i}}\right)\left(\frac{\mu}{\rho}\right)_{i}\right)}$

$N_{e l}=\frac{N}{\sum_{i} n_{i} A_{i}} Z_{e f f} \sum_{i} n_{i}$

where $n_{i}$ is the number of atoms of $i$ th element in a given molecule, $(\mu / \rho)_{\text {bio }}$ the mass attenuation coefficient of biomolecule, $N$, the Avogadro's number, $A_{i}$, the atomic weight of element $i$. $(\mu / \rho)_{b i o}$ was estimated based on the chemical composition $\left[\left(\mathrm{Ca}_{10}\left(\mathrm{PO}_{4}\right)_{6}(\mathrm{OH})_{2}\right)\right]$ and $f_{i}$ is the molar fraction. The nominator in the equation (2) gives atomic crosssection and denominator of this equation gives electronic crosssection. Similarly $Z_{e f f}$ and $N_{e l}$ of cortical bone are computed based on their composition (ICRU-44). The computed $Z_{\text {eff }}$ and $N_{e l}$ are given in Figs. 1 and 2.

\subsection{Computation of GP fitting parameters}

We have evaluated the GP fitting parameters $\left(b, c, a, X_{k}\right.$ and $\left.d\right)$ using following expression which is based on Lagrange's interpolation technique:

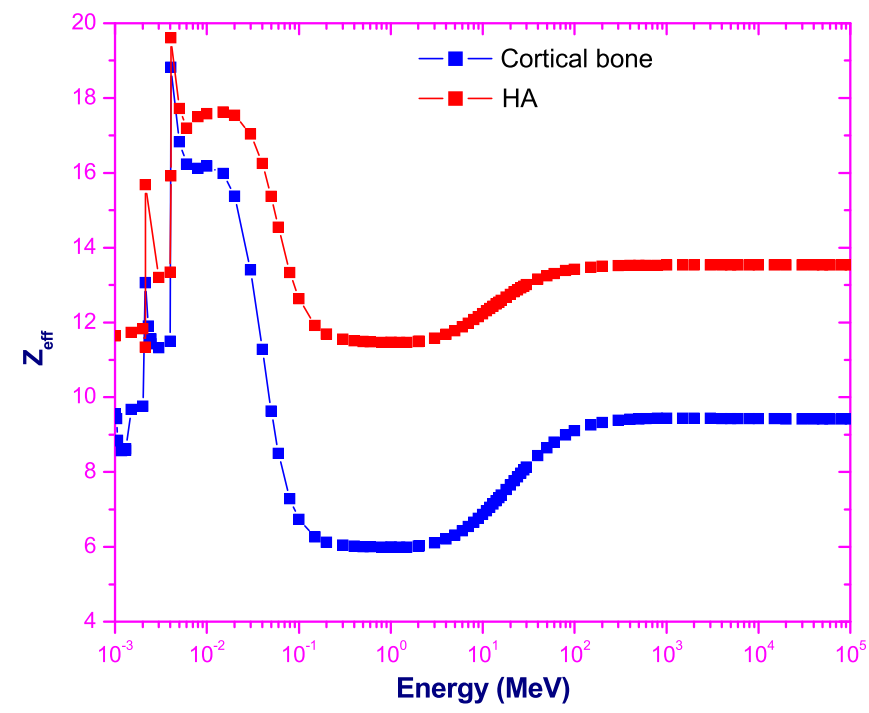

Fig. 1. Variation of effective atomic number $Z_{\text {eff }}$ of hydroxyapatite and cortical bone with photon energy for total photon interaction. 


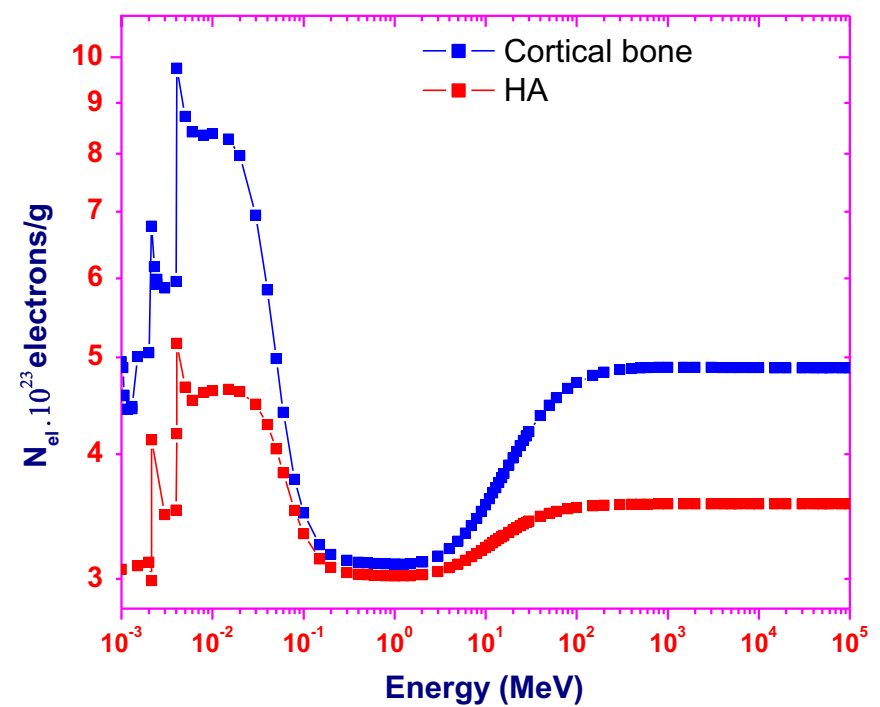

Fig. 2. Variation of effective electron density $N_{e l}$ of hydroxyapatite and cortical bone with photon energy for total photon interaction.

$P_{Z_{\text {eff }}}=\sum\left(\frac{\prod_{Z^{\prime} \neq Z}\left(Z_{\text {eff }}-Z\right)}{\prod_{z \neq Z}(z-Z)}\right) P_{Z}$

where lower case $z$ is the atomic number of the element of known GP fitting parameter $P_{z}$ adjacent to the effective atomic number $\left(Z_{\text {eff }}\right)$ of the given material whose GP fitting parameter $P_{Z_{\text {eff }}}$ is desired and upper case $Z$ are atomic numbers of other elements of known GP fitting parameter adjacent to $Z_{\text {eff. }}$ GP fitting parameters $\left(b, c, a, X_{k}\right.$ and $d$ ) for element adjacent to $Z_{\text {eff }}$ are provided by the standard data available in literature [ANSI/ANS 6.4.3 (1991)]. The computed energy absorption and exposure GP fitting parameters for HA is given in Table 1 .

\subsection{Computation of build-up factors}

The computed GP fitting parameters ( $b, c, a, X_{k}$ and $d$ ) were then used to compute the energy absorption and exposure build-up factors in the energy range $0.015 \mathrm{MeV}-15 \mathrm{MeV}$ up to a penetration depth of 40mean free path with the help of GP fitting formula, as given by the equations:

$B(E, X)=1+\frac{b-1}{K-1}\left(K^{X}-1\right)$ for $K \neq 1$

$B(E, X)=1+(b-1) X \quad$ for $K=1$
$K(E, X)=C X^{a}+d \frac{\tanh \left(\frac{X}{X_{K}}-2\right)-\tanh (-2)}{1-\tanh (-2)}$

where $X$ is the source-detector distance for the medium in mean free paths (mfp) and $b$ is the value of exposure build-up factor at $1 \mathrm{mfp} . K(E, X)$ is the dose multiplication factor and $b, c, a, X_{k}$ and $d$ are computed GP fitting parameters that depend on attenuating medium and source energy.

\section{Results and discussions}

The variation of $Z_{\text {eff }}$ and $N_{e l}$ with photon energy for total photon interactions are as shown in Figs. 1 and 2 and this variation is because of dominance of different photon interactions. In lower energy region, photoelectric interaction dominates, hence $Z_{\text {eff }}$ varies similar to photoelectric absorption. There is a slight increase in the $Z_{\text {eff }}$ up to $15 \mathrm{keV}$ and becomes maximum then decreases sharply. It remains constant from $0.2 \mathrm{MeV}$ to $2 \mathrm{MeV}$ which shows that coherent and incoherent processes increases. From $2 \mathrm{MeV}$ to $300 \mathrm{MeV}$, there is regular increase in the $Z_{\text {eff }}$ with photon energy. This is due to the increase in incoherent and pair production processes. From $300 \mathrm{MeV}$ onwards $Z_{\text {eff }}$ remains constant which is due to dominance in pair production processes. It is found that $Z_{\text {eff }}$

Table 1

GP fitting parameters of HA.

\begin{tabular}{|c|c|c|c|c|c|c|c|c|c|c|}
\hline \multirow[t]{2}{*}{ Energy(MeV) } & \multicolumn{5}{|c|}{ GP fitting parameters for energy absorption build-up factors } & \multicolumn{5}{|c|}{ GP fitting parameters for exposure build-up factors } \\
\hline & $b$ & $c$ & $a$ & $X_{k}$ & $d$ & $b$ & $c$ & $a$ & $X_{k}$ & $d$ \\
\hline 0.015 & 1.010 & 0.457 & 0.171 & 26.008 & -0.262 & 1.010 & 0.457 & 0.171 & 26.008 & -0.262 \\
\hline 0.020 & 1.026 & 0.428 & 0.177 & 30.134 & -0.350 & 1.026 & 0.428 & 0.177 & 30.134 & -0.350 \\
\hline 0.030 & 1.097 & 0.364 & 0.234 & 13.661 & -0.134 & 1.097 & 0.365 & 0.234 & 13.661 & -0.134 \\
\hline 0.040 & 1.245 & 0.403 & 0.213 & 14.652 & -0.121 & 1.245 & 0.403 & 0.213 & 14.652 & -0.121 \\
\hline 0.050 & 1.538 & 0.509 & 0.164 & 15.047 & -0.089 & 1.538 & 0.509 & 0.164 & 15.047 & -0.089 \\
\hline 0.060 & 2.073 & 0.567 & 0.155 & 13.636 & -0.083 & 2.073 & 0.567 & 0.155 & 13.636 & -0.083 \\
\hline 0.080 & 3.286 & 0.891 & 0.043 & 14.070 & -0.038 & 2.780 & 0.889 & 0.045 & 13.710 & -0.018 \\
\hline 0.100 & 4.090 & 1.198 & -0.031 & 13.195 & -0.002 & 2.941 & 1.154 & -0.019 & 13.592 & 0.006 \\
\hline 0.150 & 4.094 & 1.603 & -0.105 & 13.301 & 0.038 & 3.046 & 1.464 & -0.077 & 15.048 & 0.015 \\
\hline 0.200 & 3.638 & 1.715 & -0.121 & 13.718 & 0.044 & 2.891 & 1.565 & -0.093 & 15.566 & 0.023 \\
\hline 0.300 & 3.042 & 1.733 & -0.125 & 14.034 & 0.045 & 2.626 & 1.595 & -0.099 & 15.127 & 0.025 \\
\hline 0.400 & 2.736 & 1.675 & -0.118 & 14.332 & 0.041 & 2.459 & 1.564 & -0.097 & 15.272 & 0.026 \\
\hline 0.500 & 2.545 & 1.617 & -0.111 & 14.342 & 0.039 & 2.333 & 1.527 & -0.094 & 15.414 & 0.027 \\
\hline 0.600 & 2.409 & 1.557 & -0.102 & 14.570 & 0.036 & 2.238 & 1.486 & -0.088 & 15.353 & 0.026 \\
\hline 0.800 & 2.232 & 1.460 & -0.088 & 14.841 & 0.031 & 2.109 & 1.412 & -0.078 & 15.395 & 0.024 \\
\hline 1.000 & 2.113 & 1.383 & -0.076 & 14.975 & 0.028 & 2.014 & 1.353 & -0.070 & 15.695 & 0.023 \\
\hline 1.500 & 1.938 & 1.251 & -0.054 & 14.387 & 0.020 & 1.873 & 1.234 & -0.049 & 14.889 & 0.017 \\
\hline 2.000 & 1.840 & 1.159 & -0.035 & 14.588 & 0.013 & 1.791 & 1.155 & -0.034 & 15.254 & 0.012 \\
\hline 3.000 & 1.700 & 1.056 & -0.011 & 13.026 & 0.000 & 1.678 & 1.061 & 0.003 & 13.937 & -0.001 \\
\hline 4.000 & 1.609 & 0.989 & 0.007 & 14.040 & -0.010 & 1.601 & 0.995 & 0.005 & 12.968 & -0.009 \\
\hline 5.000 & 1.548 & 0.927 & 0.027 & 12.943 & -0.025 & 1.538 & 0.944 & 0.022 & 11.277 & -0.021 \\
\hline 6.000 & 1.475 & 0.929 & 0.025 & 15.484 & -0.028 & 1.485 & 0.932 & 0.025 & 11.936 & -0.020 \\
\hline 8.000 & 1.380 & 0.909 & 0.032 & 12.115 & -0.023 & 1.404 & 0.903 & 0.034 & 13.827 & -0.028 \\
\hline 10.000 & 1.309 & 0.915 & 0.031 & 14.507 & -0.027 & 1.340 & 0.890 & 0.040 & 13.113 & -0.033 \\
\hline 15.000 & 1.229 & 0.838 & 0.061 & 14.182 & -0.055 & 1.248 & 0.859 & 0.053 & 14.300 & -0.054 \\
\hline
\end{tabular}




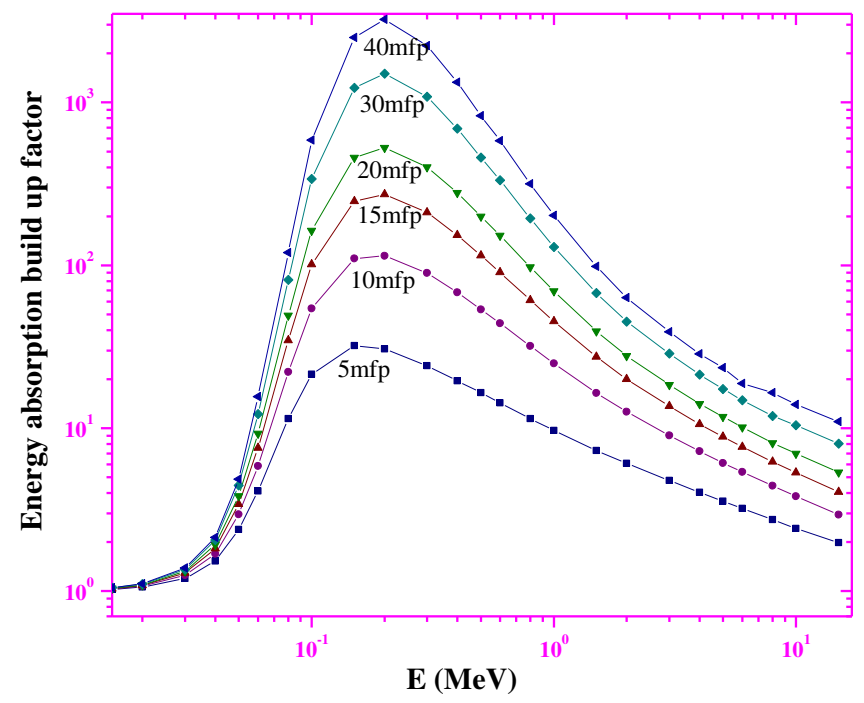

Fig. 3. Variation of energy absorption build-up factor with photon energy for hydroxyapatite.

values of HA vary from the element with lowest $Z$ to the highest $Z$ present in their composition.

The variation of energy absorption and exposure build-up factors with incident photon energy for HA is shown in Figs. 3 and 4. From these figures it is observed that energy absorption and exposure build-up factors increases up to the $E_{p e}$ and then decreases. Here $E_{p e}$ is the energy value at which the photoelectric interaction coefficients matches with Compton interaction coefficients for a given value of effective atomic number $\left(Z_{\text {eff }}\right)$. $E_{p e}$ is almost equal to $0.1 \mathrm{MeV}$ and for both exposure and absorption build-up factors. The variation of build-up factors with energy is due to dominance of photoelectric absorption in the lower end and dominance of pair production in the higher photon energy region. In the lower energy end photoelectric absorption is dominant photon interaction process; hence energy absorption build-up factor values minimum. As the energy of incident photon increases, Compton scattering overtakes the photoelectric absorption. It results multiple Compton scattering events which increases the energy absorption build-up factor up to the $E_{p e}$ and becomes

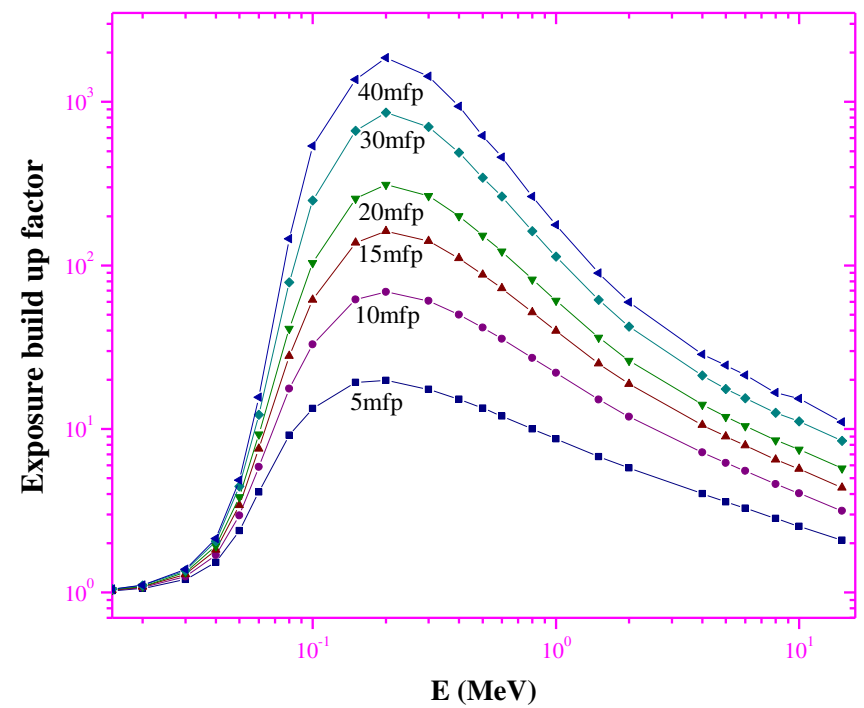

Fig. 4. Variation of exposure build-up factor with photon energy for hydroxyapatite.

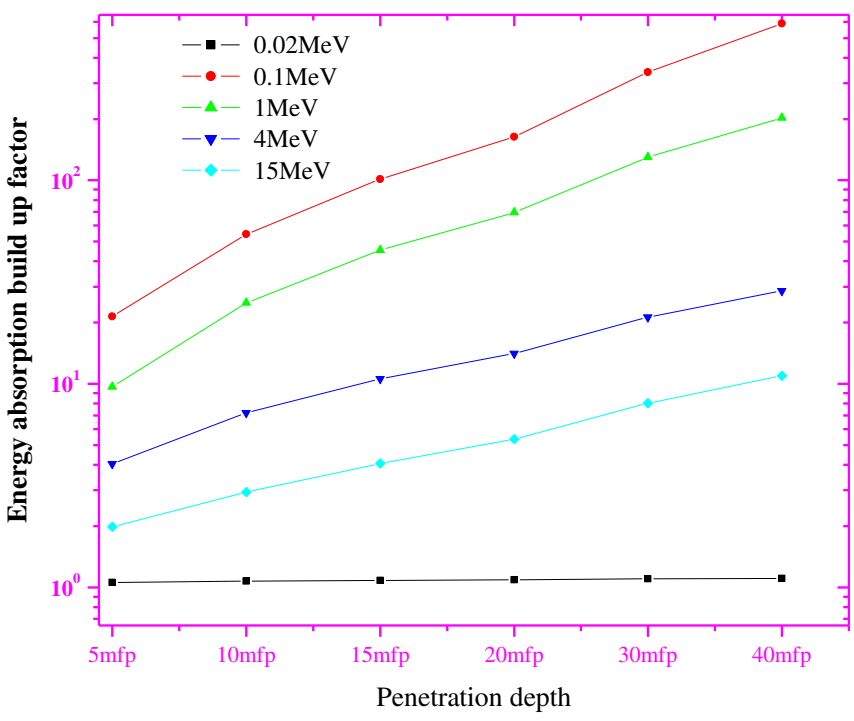

Fig. 5. Variation of energy absorption build-up factor with penetration depth for hydroxyapatite.

maximum at $E_{p e}$. Thereafter (above $E_{p e}$ ), pair production starts dominating (absorption process) which reduces the energy absorption build-up factor to a minimum value. The variation of energy absorption and exposure build-up factors with penetration depth of $\mathrm{HA}$ at $0.02 \mathrm{MeV}, 0.1 \mathrm{MeV}, 1 \mathrm{MeV}, 4 \mathrm{MeV}$ and $15 \mathrm{MeV}$ is shown in Figs. 5 and 6. The build-up factor of HA increases with penetration depth. With increase in penetration depth, thickness of the interacting material has been increased which results in increasing the scattering events in the interacting medium. Hence it results in large energy absorption build-up factor values. So that the degree of violation of Lambert-Beer $\left(I=I_{0} e^{-\mu t}\right)$ law is less for least penetration depth.

The specific absorbed fraction of energy $\Phi$ is calculated the using the point source kernel method. In this method, the specific absorbed fraction of energy at distance $x$ from the point source monoenergetic photon emitter is

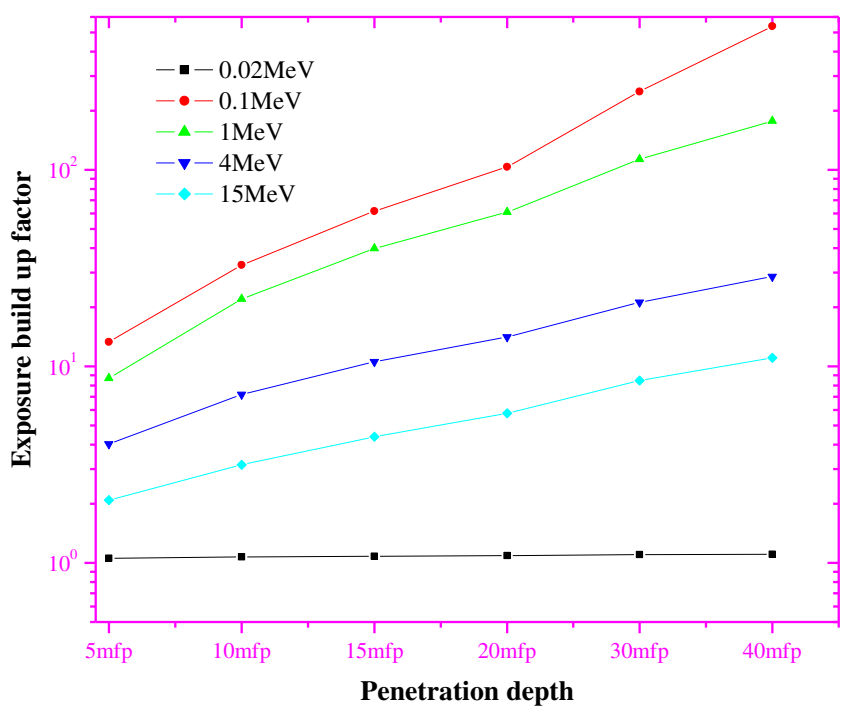

Fig. 6. Variation of exposure build-up factor with penetration depth for hydroxyapatite. 


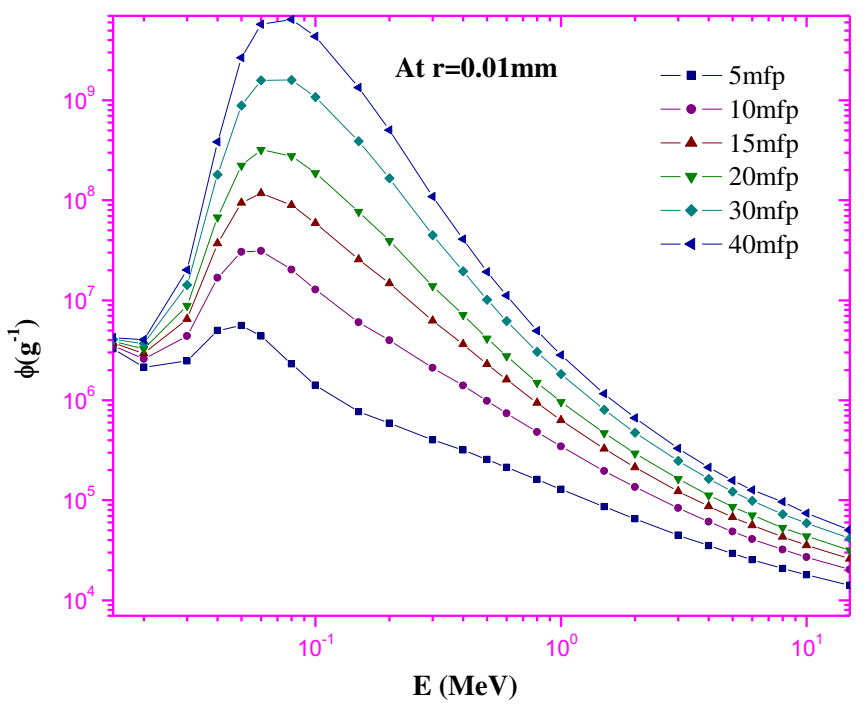

Fig. 7. The variation of specific fraction of absorbed energy $(\Phi)$ with incident photon energy in hydroxyapatite at $r=0.01 \mathrm{~mm}$ and for various penetration depths up to $40 \mathrm{mfp}$.

$\Phi(x)=\frac{\mu_{e n} \exp (\mu x) B_{e n}}{4 \pi x^{2} \rho}$

Here $\mu_{e n}$ is linear absorption coefficient of photons of given energy, $\mu$ is linear attenuation coefficient of photons of given energy, $B_{e n}$ is energy absorption build-up factor; $\rho$ is density of the medium. The computed energy absorption build-up factors may be useful to evaluate $\Phi$ which intern helps for accurate dose calculation in HA. The variation of specific absorbed fraction of energy $\left(\mathrm{g}^{-1}\right)$ in HA at $5 \mathrm{mfp}, 10 \mathrm{mfp}, 15 \mathrm{mfp}, 20 \mathrm{mfp}$ and $40 \mathrm{mfp}$ for various interacting thickness of the medium $(0.01 \mathrm{~mm}, 0.1 \mathrm{~mm}, 1 \mathrm{~mm})$ are shown in Figs. 7-9. From these figures it is observed that the specific absorbed fraction of energy $(\Phi)$ also increases up to the $E_{p e}$ $(0.1 \mathrm{MeV})$ and then decreases. The variation of $\Phi$ with energy is due to dominance of photoelectric absorption in the lower end and

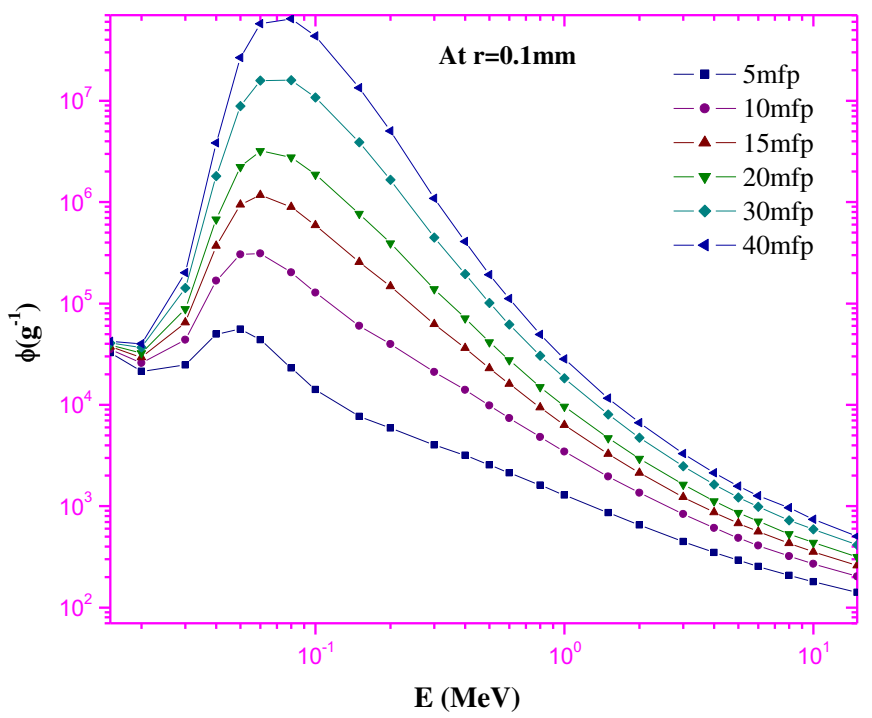

Fig. 8. The variation of specific fraction of absorbed energy $(\Phi)$ with incident photon energy in hydroxyapatite at $r=0.1 \mathrm{~mm}$ and for various penetration depths up to $40 \mathrm{mfp}$.

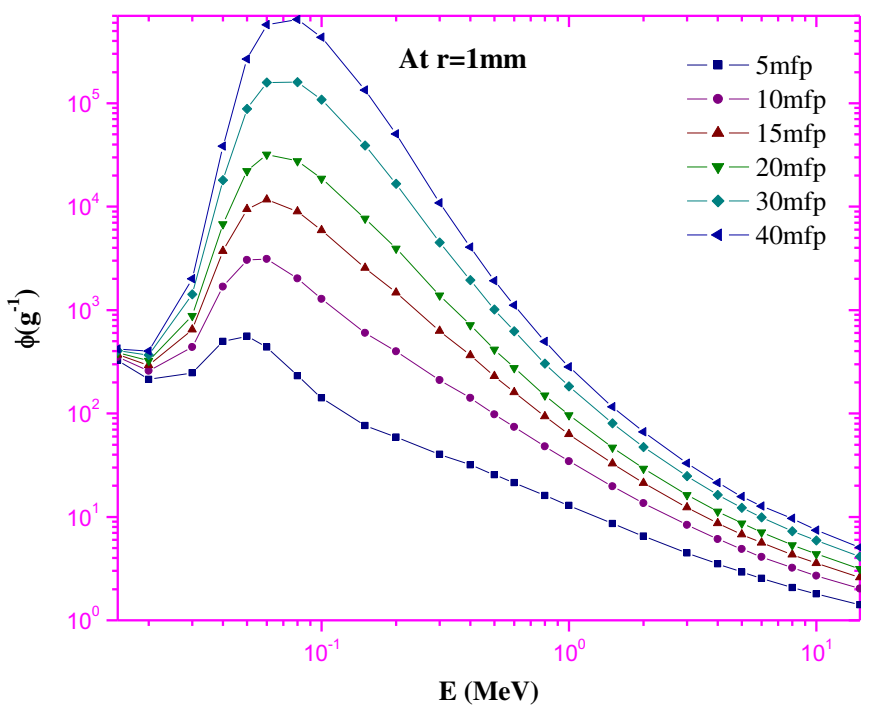

Fig. 9. The variation of specific fraction of absorbed energy $(\Phi)$ with incident photon energy in hydroxyapatite at $r=1 \mathrm{~m}$ and for various penetration depths up to $40 \mathrm{mfp}$.

dominance of pair production in the higher photon energy region. In the lower energy end photoelectric absorption is dominant photon interaction process; hence $\Phi$ values minimum. As the energy of incident photon increases, Compton scattering overtakes the photoelectric absorption. It results multiple Compton scattering events which increases the value of $\Phi$ up to the $E_{p e}$ and becomes maximum at $E_{p e}$. Thereafter (above $E_{p e}$ ), pair production starts dominating (absorption process) which reduces the value of $\Phi$ to minimum. The variation of specific absorbed fraction of energy $\left(\mathrm{g}^{-1}\right)$ of HA with penetration depth for various interacting thickness of the medium (0.01 $\mathrm{m}$ and $0.1 \mathrm{~m})$ at $0.01 \mathrm{MeV}, 0.1 \mathrm{MeV}, 10 \mathrm{MeV}$, and $15 \mathrm{MeV}$ is shown in Figs. 10 and 11. The specific absorbed fraction of energy for HA increases with penetration depth. With increase in penetration depth, thickness of the interacting material has been increased which results in increasing the scattering events in the interacting medium. Hence it results in large $\Phi$ values. The

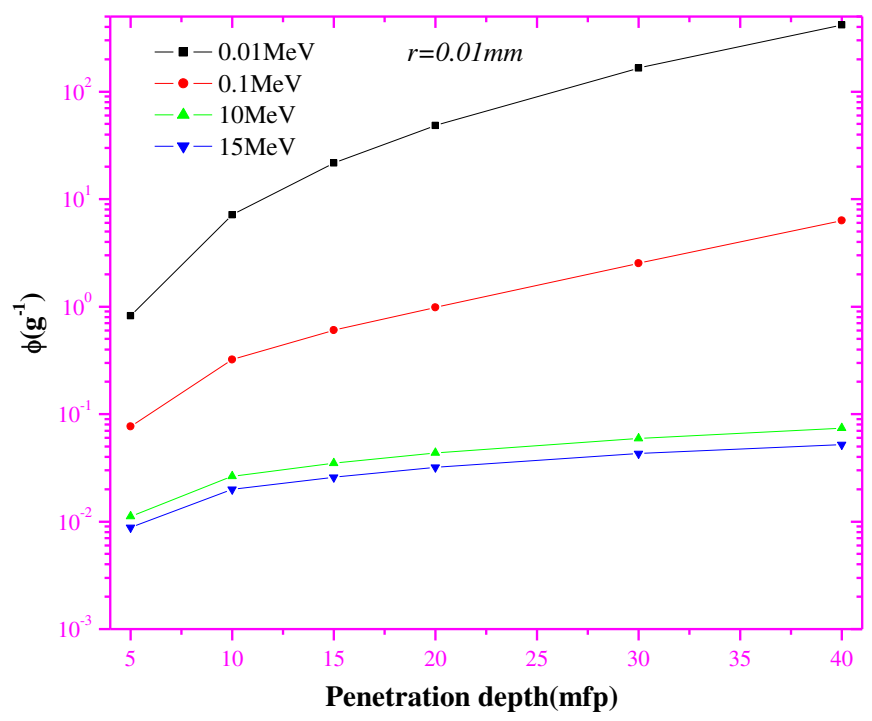

Fig. 10. The variation of specific fraction of absorbed energy $(\Phi)$ with penetration depth in hydroxyapatite at $r=0.01 \mathrm{~mm}$ and for various incident energies up to $15 \mathrm{MeV}$. 


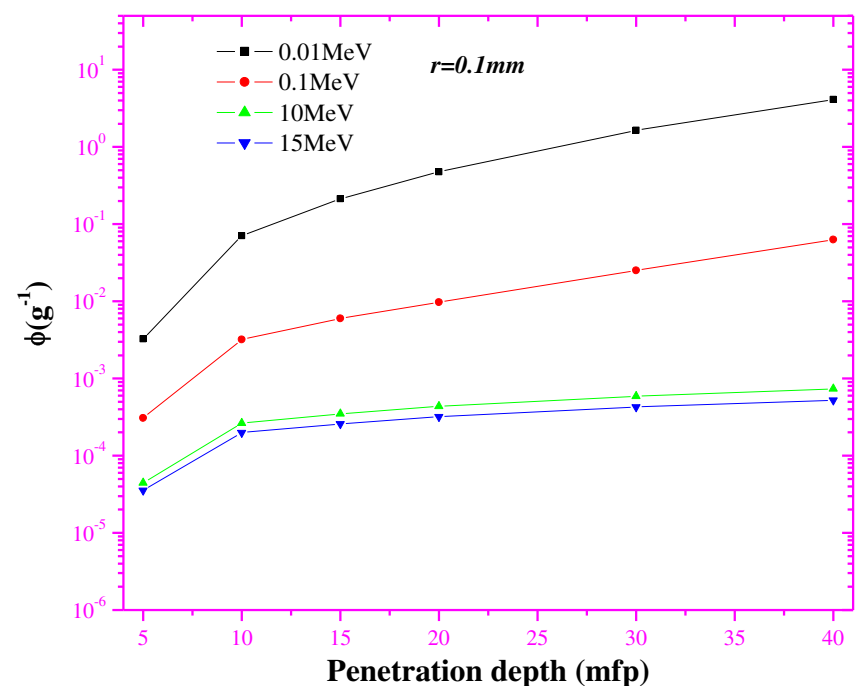

Fig. 11. The variation of specific fraction of absorbed energy $(\Phi)$ with penetration depth in hydroxyapatite at $r=0.1 \mathrm{~mm}$ and for various incident energies up to $15 \mathrm{MeV}$.

specific absorbed fraction of energy decreases with the increase of thickness of the interacting medium.

The radial dependence of dose can be represented by exp $(-\mu r)$ $B / r^{2}$. Here $\mu$ is denotes the linear attenuation coefficient for the appropriate photon energy and $B$ is an absorption build-up factor. Hence photon dose at a distance $r$ is given by $D_{r}=D_{0} \exp (-\mu r) B / r^{2}$. Here $D_{0}$ is initial dose delivered by the point gamma ray emitter The relative dose is $D_{r} / D_{0}=\exp (-\mu r) B / r^{2}$. Hence relative dose of photon in HA is calculated from the computed absorbed build-up factor. The product of $D_{0}$ and relative dose gives actual photon dose at a distance $r$. The variation of relative dose $\left(D_{r} / D_{0}\right)$ with photon energy in HA at $40 \mathrm{mfp}$ for various distances $(r=0.01 \mathrm{~m}$ to $1 \mathrm{~m})$ is shown in Fig. 12. From this figure it is observed that relative dose $\left(D_{r} / D_{0}\right)$ also increases up to the $E_{p e}$ and then decreases. The variation of relative dose $\left(D_{r} / D_{0}\right)$ with distance $(r)$ in HA at $40 \mathrm{mfp}$ for various energies is shown in Fig. 13. Calculation of the dose distribution around point gamma ray emitters is useful in clinical dosimetry.

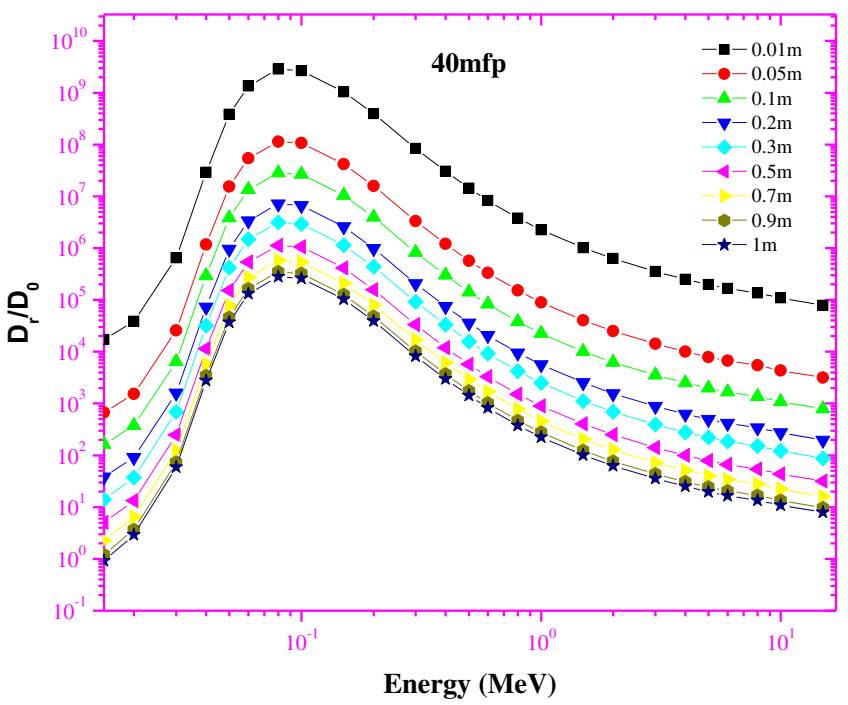

Fig. 12. Variation of relative dose $\left(D_{r} / D_{0}\right)$ with photon energy in HA at $40 \mathrm{mfp}$ at various distances.

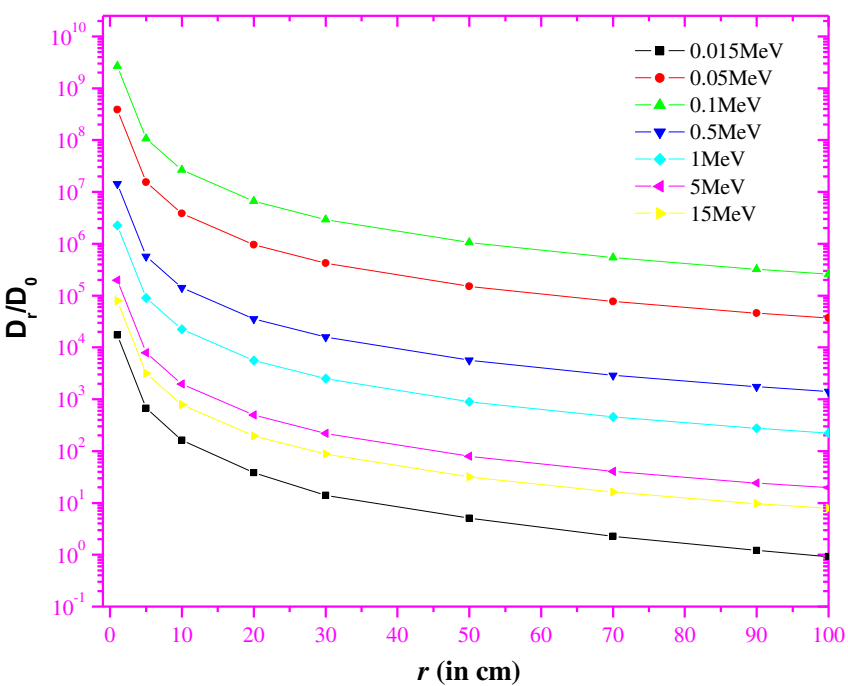

Fig. 13. Variation of relative dose $\left(D_{r} / D_{0}\right)$ with distance $(r)$ in hydroxyapatite at $40 \mathrm{mfp}$ at various energies.

Phantoms are constructed from materials having good tissue equivalence with respect to absorption of ionizing radiation. The fundamental advantage of such materials is that they allow the absorbed dose to be determined when information on the energy and nature of the charged particles at the point of interest is incomplete. For a given radiation type and energy, these materials should absorb and scatter radiation to the same extent, within acceptable limits, as irradiated tissue. Hydroxyapatite (HA) has been applied as filling material for bone defects and augmentation, artificial bone graft material, and prosthesis revision surgery. Its high surface area leads to excellent osteoconductivity and resorbability providing fast bone in growth. It is seen that the energy behavior of the effective atomic number of cortical bone is similar to hydraxyapatite (HA). When bone/HA is exposed to X-rays and gamma rays, then the maximum radiation dose to the tissue may not be at its surface but somewhere inside, due to the build-up of degraded photons by multiple scattering within the tissue. With proper knowledge of build-up factors of bone/HA, energy absorption can be carefully controlled. The results of the present paper will also help in estimating safe dose levels for radiotherapy patients and also will be useful in dosimetry and diagnostics.

\section{References}

Agarwala, S., Bhagawat, A., 2005. Hydroxyapatite as bone graft substitute: use in cortical and cacellous bone. Indian J. Orthop. 39 (4), 254-256.

American National Standard (ANS), 1991. Gamma-ray Attenuation Coefficients and Buildup Factor for Engineering Materials. ANSI/ANS 6.4.3.

Aoki, S., Yamaghuci, S., Nakahira, A., Suganuma, K., 2004. Preparation of porous calcium phosphates using a ceramic foaming technique combined with a hydrothermal treatment and the cell response with incorporation of osteoblast-like cells. J. Ceram. Soc. Japan 112 (130), 193-199.

Asano, Y., Sakamoto, Y., 2007. Gamma-ray Buildup Factors for Heavy Concretes. JAEData/Code 2007-006. Japan Atomic Energy Agency, Ibaraki-ken, Japan.

Banfi, A., Muraglia, A., Dozin, B., Mastrogiacomo, M., Cancedda, R., Quarto, R., 2000. Proliferation kinetics and differentiation potential of ex vivo expanded human bone marrow stromal cells: implications for their use in cell therapy. Exp. Hematol. 28 (6), 707-715.

Byrne, R.S., Deasy, P.B., 2002. Use of commercial porous ceramic particles for sustained drug delivery. J. Inter. Pharm. 246, 61-73.

Damien, E., Revell, P.A., 2004. Coralline hydroxyapatite bone graft substitute: a review of experimental studies and biomedical applications. J. Appl. Biomater. Biomech. 2 (2), 65-73.

Eisenhauer, C.M., Simmons, G.L., 1975. Point isotropic gamma-ray buildup factors in concrete. Nucl. Sci. Eng. 56, 263-270.

Frieb, W., Warner, J., 2002. In: Schuth, F., Sing, K.S.W., Weitkamp., J. (Eds.), Handbook of Porous Solids. Wiley-VCH, Weinheim, pp. 2923-2953. 
Gerward, L., Guilbert, N., Jensen, K.B., Levring, H., 2004. WinXCom - A program for calculating X-ray attenuation coefficients. Radiat. Phys. Chem. 71, 653-654.

Gopinath, D.V., Samthanam, K., 1971. Radiation transport in one-dimensional finite systems part I. Development in the anisotropic source - flux technique. Nucl. Sci. Eng. 43, 186.

Harima, Y., Sakamoto, Y., Tanaka, S., Kawai, M., 1986. Validity of the geometricprogression formula in approximating the gamma ray buildup factors. Nucl. Sci. Eng. 94, 24-30.

Harima, Y., 1993. An historical review and current status of buildup factor calculations and applications. Radiat. Phys. Chem. 41 (4-5), 631-672.

ICRU, 1989. Tissue Substitutes in Radiation Dosimetry and Measurement. Report No. 44. Hench, L.L., 1998. Biomaterials: a forecast for the future. Biomaterials 19, 1419-1423.

Komlev, V.S., Barinov, S.M., Girardin, E., Oscarsson, S., Rosengren, A., Rustichelli, Orlovskii, V.P., 2003. Porous spherical hydroxyapatite and fluorhydroxyapatite granules: processing and characterization. Sci. Technol. Adv. Mater. 4(6), 503-508.

Krajewski, A., Ravaglioli, A., Roncari, E., Pinasco, P., 2000. Porous ceramic bodies for drug delivery. J. Mater. Sci. Mater. Med. 12, 763-771.

Manjunatha, H.C., Rudraswamy., B., 2011a. Computation of exposure build-up factors in teeth. Radiat. Phys. Chem. 80, 14

Manjunatha, H.C., Rudraswamy., B., 2011b. A study of thickness and penetration depth dependence of specific absorbed fraction of energy in bone. Ann. Nucl. Energy 38, 2271-2282.

Muraglia, A., Cancedda, R., Quarto, R., 2000. Clonal mesenchymal progenitors from human bone marrow differentiate in vitro according to a hierarchical model. J. Cell Sci. 113, 1161-1166.
Moore, W.R., Graves, S.E., Bain, G.I., Aust, N.Z., 2001. Synthetic bone graft substitutes. J. Surg. 71, 354-361.

Ohgushi, H., Caplan, A.I., 1999. Stem cell technology and bioceramics: from cell to gene engineering. J. Biomed. Mater. Res. 48, 913-927.

Ryman, J.C., Alpan, F.A., Durani, L.A., et al., 2008. Revision of ANSI/ANS-6.4.3. Trans. Am. Nucl. Soc. 99, 613-614.

Ruggieri, Sanders, 2008. Trans. Am. Nucl. Soc. 99, 618-620.

Sopyan, I., Mel, S., Ramesh, K.A., 2007. Khalid porous hydroxyapatite for artificial bone applications. Sci. Technol. Adv. Mater. 8, 116-123.

Singh, S.S., Singh, Tejbir, Kaur, Paramajeet, 2008. Variation of energy absorption build-up factors with incident photon energy and penetration depth for some commonly used solvents. Ann. Nucl. Energy 35, 1093-1097.

Simmons, G.L., 1973. An Adjoint Gamma-ray Moments Computer Code ADJMOM-1. National Bureau of Standards Technical Note 748.

Shimizu, A., Onda, T., Sakamoto, Y., 2004. Calculations of gamma-ray buildup factor up to depths of $100 \mathrm{mfp}$ by the method of invariant embedding, (III) generation of an improved data set. J. Nucl. Sci. Technol. 41, 413-418.

Takeuchi, K., 1973. PALLAS-PL, SP-A Code Dimensional Transport Code, vol. 42. Ship Research Institute, Japan.

United States Patent 6210715, Calcium Phosphate Microcarriers and Microspheres, 2001.

Yamano, N., Minami, K., Koyama, K., Naito, Y., 1989. RADHEAT V-4: a Code System to Generate Multigroup Constants and Analyse Radiation Transport for Shielding Safety Evaluation. Japan Atomic Energy Research Institute. Report 1316. 